\title{
Secado de Pulpa de Café: Condiciones de Proceso, Modelación Matemática y Efecto sobre Propiedades Fisicoquímicas
}

\author{
Laura S. Torres-Valenzuela ${ }^{1(*)}$, Katherine G. Martínez ${ }^{1}$, Johanna A. Serna-Jimenez ${ }^{1}$ y María C. Hernández ${ }^{1}$ \\ (1) Universidad La Gran Colombia Seccional Armenia, Facultad de Ingenierías, Programa de Ingeniería \\ Agroindustrial, km 7 vía Armenia - La Tebaida, Armenia, Quindío, Colombia. \\ (e-mail: torresvallaura@miugca.edu.co; sernajimjohanna@miugca.edu.co; \\ martinezcorkatherine@miugca.edu.co; hernandezsanmariacamila@miugca.edu.co)
}

${ }^{*}$ Autor a quien debe ser dirigida la correspondencia.

Recibido Ago. 7, 2018; Aceptado Oct. 10, 2018; Versión final Nov. 28, 2018, Publicado Abr. 2019

\section{Resumen}

El objetivo de este trabajo fue evaluar las condiciones de secado de cáscara de café y su efecto sobre propiedades fisicoquímicas. Se realizaron cinéticas de secado hasta 420 minutos, a 40,50 y $60 \stackrel{\circ}{ } \mathrm{C}$; los datos experimentales se ajustaron con 4 modelos cinéticos de capa delgada. Adicionalmente, se evaluó el contenido de cafeína y ácido clorogénico y los parámetros de color en la cáscara húmeda y seca. Se obtuvo una reducción del contenido de agua hasta el 13,5\%. El modelo de Page fue el más adecuado para representar el secado a las tres temperaturas evaluadas. Respecto a los biocomponentes, la concentración se incrementó en el proceso y los cambios de color fueron superiores al $4 \%$, generando tonalidades rojizas y cambios en el ángulo de tono, luminosidad y croma. Lo anterior demuestra que el secado es una alternativa para estabilizar este subproducto, como etapa previa para su uso industrial.

Palabras clave: ácido clorogénico; cafeína; color; modelos cinéticos; subproductos

\section{Drying of Coffee Pulp: Process Parameters, Mathematical Model and its Effect over Physicochemical Properties}

\begin{abstract}
The main objective of this work it is to evaluate the husk's drying process and its effect over the husk physicochemical properties. The kinetics of the drying process was followed during 420 minutes using temperatures of 40,50 and $60^{\circ} \mathrm{C}$. The experimental data matches 4 kinetics models of thin husk. Additionally, caffeine and chlorogenic acid content was evaluated along with the color parameters of humid and dry husk. Water content reduction of $13 \%$ was obtained. Page's model was the best one to describe the drying process at the three temperatures. Regarding the biocompounds, its value was incremented by $4 \%$, generating in this way reddish tonalities and changes in the tone angle, luminosity and chroma. These results evidence that drying process is an alternative to stabilize this byproduct, in a previous step before its industrial use.
\end{abstract}

Keywords: chlorogenic acid; caffeine; color; kinetic models; byproducts 


\section{INTRODUCCIÓN}

El café es uno de los productos de mayor comercialización a nivel mundial, es cultivado en más de 80 paĺses, de los cuales el $90 \%$ son considerados en vía de desarrollo (Dadi et al., 2018). Se estima que el $80 \%$ de la población adulta ha consumido bebidas a base de café, por lo cual se considera a segunda bebida más consumida después del agua (Hall et al., 2015); diariamente se consumen aproximadamente 1,6 billones de tazas de café en el mundo (Cappelletti et al., 2015). Esta cifra que va en aumento considerando el efecto positivo que causa su ingesta en la salud, debido a la composición bioquímica característica de éste (Komes y Bušić, 2014). El café posee componentes químicos, entre los que se destacan sustancias orgánicas como carbohidratos, lípidos, proteínas, alcaloides (cafeína) y trigonelina, así como, ácidos carboxílicos, fenólicos y compuestos volátiles responsables del aroma (Toci y Boldrin, 2018). Todos en conjunto dan como resultado una gran diversidad y complejidad de estructuras (Montilla-Pérez, et al., 2008) y variabilidad dependiendo de la especie (Puerta, 2011). En el caso de Coffea arábica tiene un mayor contenido de lípidos y de sacarosa que Coffea canephora. La Robusta se diferencia por su mayor contenido de polisacáridos, cafeína, ácidos clorogénicos y cenizas. Adicionalmente, el café se ha considerado como una fuente rica de antioxidantes de la familia de ácidos hidroxicinámicos (cafeico, clorogénico, cumarico, ferulico y sinapínico) y otros compuestos biológicamente activos con alto potencial antioxidante, como la cafeína, ácido nicotínico, trigonelina, cafestol y kahweol (Komes y Bušić, 2014).

Los ácidos clorogénicos son una serie de ésteres fenólicos que se derivan de la unión de un éster entre el ácido cafeico y el ácido quínico (Gotteland y De Pablo, 2007). El contenido de ácidos clorogénicos es del $7,0 \%$ en el café verde y después del tostado alcanza un contenido del 4,0\% (Gotteland y De pablo 2007). Este compuesto tiene una capacidad antioxidante significativa y además es estimulante, expectorante, diurético, colerético y antihepatotóxico (Fonnegra y Silvia, 2007). Otro biocomponente de importancia es la cafeína, catalogada como una de las sustancias psicoactivas más ingeridas en el mundo (Burdan, 2015). La cafeína es una metilxantina compuesto perteneciente a la familia de los alcaloides, a estos compuestos se les atribuye múltiples beneficios a la salud, pues actúa en el sistema nervioso central, cardiovascular, respiratorio, renal y muscular (National Academy of Science, 2001). Estos biocomponentes también se encuentran presentes en la cáscara de café. La comercialización del café se realiza como café verde. El proceso de transformación inicia cuando el material vegetal (fruto maduro) es recolectado, y se transforma a café pergamino seco. El proceso se realiza en el menor tiempo posible, teniendo en cuenta que este producto es perecedero debido a la acelerada fermentación ocasionada por el contenido de agua y azúcares presentes en el grano y la cáscara (Rodríguez, et al., 2015). En este proceso se aprovecha menos del $5 \%$ de la biomasa generada en la bebida (Rodríguez y Zambrano, 2010), el resto queda de manera residual como hojas, ramas, frutos verdes, pulpa, mucilago, borra, entre otros.

El principal método de transformación (para obtener café verde), es denominado beneficio húmedo. En este proceso la cascara del café es removida, posteriormente se elimina el mucílago y finalmente el café es secado (Novita, 2016). Estas dos capas (cáscara y mucílago) constituyen aproximadamente el $56 \%$ y en su proceso de descomposición provocan malos olores y proliferación de dípteros, además los componentes químicos que estos poseen alteran las fuentes hídricas y a su vez las condiciones de vida de los organismos que viven en este medio (Armas et al., 2008). La cáscara (también denominada pulpa), corresponde aproximadamente al $43,58 \%$ del peso del fruto seco (Rodríguez y Zambrano, 2010) y contiene alrededor de un $86 \%$ de agua (Armas et al., 2008); está compuesta por el epicarpio y parte del mesocarpio del fruto. La pulpa contiene cantidades importantes de cafeína la cual representa cerca del $1.3 \%$ de su peso seco, además de otros componentes. Éstos dos subproductos del café son ricos en carbohidratos, proteínas, pectinas, compuestos bioactivos como polifenoles, convirtiéndolos en fuentes potenciales de agroindustrialización y a su vez son recursos renovables económicos a los cuales se les puede dar un alto valor agregado (Murthy y Naidu, 2012).

Actualmente para uso de estos residuos se elabora ensilaje para la alimentación animal, torta de pulpa de café, jugo tratado con microorganismos para el consumo animal (Noriega et al., 2009), adicionalmente han sido utilizados para cultivo de hongos (Murthy y Manonmani, 2008), producción de enzimas (Buntić et al., 2016; Ngo y Phan, 2016; Torres-Mancera et al., 2011), biocombustibles (Gouvea et al., 2009; Woldesenbet, Woldeyes, y Chandravanshi, 2016) y compostaje (Hachicha et al., 2012). Teniendo en cuenta que la pulpa de café es altamente perecedera debido a su alto contenido de humedad, se generan pérdidas por deterioro químico y microbiano (Ruiz-López et al., 2011). Entre los métodos de preservación, el secado resulta ser uno de los más atractivos, debido a su versatilidad, costo relativamente bajo, y a las características físicas del producto (Mahn et al., 2017). El secado es uno de los más antiguos y comunes métodos usados para la preservación de alimentos (Norhashila, et al., 2014) y el secado convectivo con aire es el método más empleado para el secado de materiales agrícolas (Motevali et al., 2014; Nuñez et al., 2016), en este proceso el aire calienta la superficie del producto, produciendo la evaporación del agua y un incremento en la temperatura, como consecuencia dentro del alimento se genera una conducción de calor y difusión del agua (García-Alvarado et al., 2014). Existen diversos factores que influyen en los procesos de secado, por lo cual 
la aplicación de métodos de simulación y/o modelación es una herramienta eficiente para evaluar el procesamiento en la industria alimentaria (Nuñez et al., 2016). Por lo anterior, los objetivos de este trabajo fueron: i) establecer el efecto del tiempo y temperatura sobre la cinética de secado de pulpa de café; ii) evaluar modelos cinéticos de capa delgada en el proceso de secado y iii) determinar los cambios en propiedades químicas y físicas en la pulpa de café por efecto del proceso de secado.

\section{MATERIALES Y MÉTODOS}

La metodología se presenta en siete subsecciones: en la primera se describe el material utilizado además de las condiciones a las cuales fue manejado; en la segunda subsección se presentan las condiciones de tiempo y temperatura para llevar a cabo el proceso de secado; la determinación de la velocidad de secado se describe en la tercera sección; para el caso de la modelación matemática de capa delgada se presenta en la cuarta sección; posteriormente se realiza la medición de las propiedades químicas y color y por último se puede observar el diseño experimental utilizado dentro de la investigación.

\section{Material vegetal}

El proceso experimental se llevó a cabo en la ciudad de Armenia, Quindío, Colombia (4²9’5" latitud norte y $75^{\circ} 44^{\prime} 49^{\prime \prime}$ longitud oeste, $1500 \mathrm{msnm}$ ) a temperatura promedio de 25 ㄷ y Humedad relativa del $75 \%$. Se trabajó con pulpa de café recién extraída por el método de beneficio Ecomill® de variedad castillo, obtenidas de un centro de acopio comunitario de la Asociación de productores de café Cuchilla del San Juan en el departamento de Risaralda, Colombia. Las muestras fueron transportadas a temperatura de $25{ }^{\circ} \mathrm{C}$. Posteriormente fueron empacadas en bolsas de polietileno de baja densidad resellables de $(25 \times 35 \mathrm{~cm}) \mathrm{calibre}$ 200 y almacenadas $\mathrm{a}-13,7^{\circ} \mathrm{C}$ hasta iniciar el proceso de secado. Es importante resaltar que el método de conservación de la materia orgánica fue un sistema de congelación lenta, con el fin de conservar y garantizar el uso de la misma durante toda la investigación, puesto que el congelar un producto retarda los cambios físicos, químicos y microbiológicos, a los cuales se les atribuye el deterioro de los alimentos.

Previo a iniciar el proceso experimental, se realizó una descongelación lenta a temperatura de $25^{\circ} \mathrm{C}$, para alcanzar un equilibrio térmico. Este proceso se realizó bajo estas condiciones con la finalidad de asemejar las características iniciales a las que se encontraba la pulpa. Sin embargo, es necesario tener en cuenta que este tipo de cambios de temperatura puede generar daños estructurales y la pérdida de componentes celulares, lo cual se manifiesta en forma de exudado en donde diversos compuestos de valor nutricional no son aprovechables.

\section{Evaluación de las condiciones de secado}

Debido a que la pulpa tiene un alto contenido de humedad (superior al $80 \%$ ) y actividad de agua superior a 0.6 , es propensa a ataque microbiano y reacciones bioquímicas de deterioro, por esta razón se determinaron las condiciones para el proceso de secado. Se realizaron curvas de secado a partir de la pulpa de café descongelada. El secado se realizó a temperaturas de 40,50 y $60^{\circ} \mathrm{C}$ y tiempos de $0,5,10,15,30,45,60,90$, 120, 180, 300 y 420 minutos. El proceso se realizó en una estufa de circulación forzada (Binder, Alemania). En cada uno de los tiempos se determinó el contenido de humedad siguiendo el método oficial AOAC 925.10 (Association of Analyitical chemists, 1990) fundamentado en gravimetría, empleando una balanza analítica (Pionner, China).

\section{Velocidad de secado}

Para determinar la velocidad de secado se establecieron los valores de la relación de peso del sólido seco (Wss) por la cantidad de agua total (Wa) contenida en el material vegetal en función del tiempo para establecer así, las condiciones de secado constante (Xt) (Geankoplis, 1998), empleando la Ecuación (1).

$$
\mathrm{Xt}=\frac{\text { Wa Kg totales de agua }}{\text { Wss Kg totales sólido seco }}
$$

Una vez obtenidos los valores correspondientes a Xt se determinaron los valores del contenido de humedad de equilibrio para cada intervalo de tiempo, representada por $X^{*}$. Con base en estos resultados se procede a definir la cantidad de humedad libre $(X)$, es decir la diferencia entre las condiciones de secado respecto a la humedad en equilibrio (Ecuación (2)).

$$
X=X t-X^{*}
$$


Al representar estos datos, se trazó una gráfica del contenido de humedad libre $(X)$ en función del tiempo (t), la cual permite establecer los valores de los cambios de humedad y tiempo, y de esta manera, determinar la velocidad de secado teniendo en cuenta los $\mathrm{Kg}$ del sólido seco (Ls) y el área superficial del material expuesta al proceso de secado (A) (Ver Ecuación (3)). Estos valores fueron graficados con el fin de obtener curva de velocidad de secado para condiciones de secado constante y determinar los periodos de secado dentro del proceso.

$$
\mathrm{R}=-\frac{\mathrm{Ls}}{\mathrm{A}} \frac{\mathrm{dX}}{\mathrm{dt}}
$$

\section{Modelación matemática}

Para determinar el modelo que mejor describe el comportamiento cinético en el proceso de secado, se evaluó el ajuste de cuatro modelos matemáticos (Tabla 1). Los parámetros de los modelos se obtuvieron mediante regresión no lineal empleando el software SPSS®. Se seleccionó el modelo de mejor ajuste a partir de la raíz del error cuadrático medio (RMSE) (Ecuación (4)), y el coeficiente de determinación $\left(R^{2}\right)$. Es importante mencionar que un error inferior al 10\% indica buena bondad de ajuste (Navia et al., 2011).

Tabla 1: Modelos cinéticos de capa delgada

\begin{tabular}{|l|l|l|}
\hline \multicolumn{1}{|c|}{ Nombre } & \multicolumn{1}{c|}{ Ecuación } & \multirow{2}{*}{ Referencia } \\
\hline Newton & $\mathrm{Y}=\exp (-\mathrm{kt})$ & (Montes, et al., 2008) \\
\hline Page & $\mathrm{Y}=\exp \left(-\mathrm{kt}^{\mathrm{n}}\right)$ & \\
\hline Henderson y Pabis & $\mathrm{Y}=\mathrm{a}[\exp (-\mathrm{kt})]$ & $\mathrm{Y}=\mathrm{a}[\exp (-\mathrm{kt})]+(1-\mathrm{a})[\exp (-\mathrm{kbt})]$ \\
\hline Difusión & $\begin{array}{l}\text { Y: Representa el contenido de humedad, t es el tiempo de secado, n, a, b y k son constantes } \\
\text { propias de cada modelo }\end{array}$ \\
\hline
\end{tabular}

$$
\text { RMSE }=\left[\frac{1}{N} \sum_{i=1}^{N}\left(M_{e}-M_{p}\right)^{2}\right]^{1 / 2}
$$

Donde:

$\mathrm{MR}_{\mathrm{e}}$ : Contenido de humedad experimental

$\mathrm{MR}_{\mathrm{p}}$ : contenido de humedad modelado

$\mathrm{N}$ : número de observaciones.

\section{Medición de propiedades químicas}

Se realizó la cuantificación de cafeína, ácido clorogénico, ácido gálico y cafeico en pulpa de café fresca y sometida a proceso de deshidratación a $60^{\circ} \mathrm{C}$ por 7 horas (determinadas a partir del modelo de Page para obtener un contenido de humedad del 13\%), siguiendo metodología reportada por Park et al. (2007). Inicialmente, se realizó la extracción de los biocompuestos a partir de la pulpa húmeda y seca utilizando etanol al $70 \%$ como solvente y un proceso de asistencia por ultrasonido (Elma, Alemania) durante 5 minutos a $37 \mathrm{KHz}$ a una potencia de 100 watts.

Posteriormente los extractos se centrifugaron a $4.000 \mathrm{rpm}$ por $10 \mathrm{~min}$ en una microcentrífuga (Unico, Estados Unidos) y el sobrenadante se filtró con un filtro de celulosa de 0,2 $\mu \mathrm{m}$. La cuantificación se llevó a cabo por cromatografía líquida de alta eficiencia (HPLC), se inyectaron $20 \mu \mathrm{L}$ de la muestra al cromatógrafo (Shimadzu, Japón) con una columna C8 (Restek, Estados Unidos). La fase móvil fue $69.9 \% \mathrm{v} / \mathrm{v}$ de agua, $30 \% \mathrm{v} / \mathrm{v}$ de metanol y $0.1 \% \mathrm{v} / \mathrm{v}$ de ácido acético y fue operada en modo isocrático, con un flujo de $0,6 \mathrm{~mL} / \mathrm{min}$ a $40^{\circ} \mathrm{C}$, con un detector UV a $254 \mathrm{~nm}$.

El análisis cuantitativo se realizó empleando soluciones estándar de cafeína y ácido clorogénico (Sigma, España), preparadas en agua ultrapura en un rango de concentraciones entre $5-100 \mu \mathrm{g} \mathrm{L}^{-1}$. La concentración de cafeína fue calculada a partir de una curva de calibración con la siguiente ecuación: y $=31024.2 \mathrm{x}-$ $34438.5\left(R^{2}=0.9980\right)$, para ácido clorogénico la ecuación es: $y=20848.1 x-8903.6\left(R^{2}=0.9996\right)$, donde y es la absorbancia (at $254 \mathrm{~nm}$ ), y x la concentración de cafeína o ácido clorogénico. 


\section{Medición de color}

El color fue medido empleando las coordenadas de color CIE-L*a*b* en un espectro de reflexión entre 400 $700 \mathrm{~nm}$, usando como referencia un observador de $10^{\circ}$ y el iluminante D65 (Cock, Valenzuela, y Aponte, 2013), empleando un colorímetro (Konica Minolta, Estados Unidos). Las coordenadas de color $L^{*} a^{*} b^{*}$ indican la luminosidad, coloración verde-rojo y coloración amarillo-azul respectivamente. Éstas fueron medidas colocando las cáscaras (antes y después del secado) en el portamuestras y se midieron en tres puntos distribuidos en forma de triángulo. La diferencia de color $(\Delta \mathrm{E})$ con respecto a la pulpa fresca fue calculado utilizando la ecuación 5.

$$
\Delta E=\sqrt{\left(\Delta L^{*}\right)^{2}+\left(\Delta a^{*}\right)^{2}+\left(\Delta b^{*}\right)^{2}}
$$

Donde:

$\Delta \mathrm{E}:$ Diferencia de color

$\Delta a$ : Diferencia en el parámetro a (-a: verde, +a: rojo)

$\Delta \mathrm{a}$ : Diferencia en el parámetro b (-b: azul, +b: amarillo)

Adicionalmente se calculó el croma $\left(C^{*}\right)$ y ángulo de tono $\left(H^{*}\right)$ con las ecuaciones 6 y 7 respectivamente

$$
\begin{aligned}
& C^{*}=\sqrt{a^{2}+b^{2}} \\
& H^{*}=\arctan \frac{b}{a}
\end{aligned}
$$

\section{Diseño experimental}

Se realizó un arreglo secuencial. En la primera etapa se utilizó un diseño factorial completamente aleatorio $3 \times 12$, donde los factores evaluados fueron temperatura con tres niveles: 40,50 y $60^{\circ} \mathrm{C}$ y tiempo con 12 niveles: $0,5,10,15,30,45,60,120,180,220,300,420(\mathrm{~min})$ para un total de 36 tratamientos, la variable de respuesta fue el contenido de humedad y se evaluó el ajuste de 4 modelos matemáticos. En la segunda fase se realizron dos tratamientos, siguiendo un diseño unifactorial con dos niveles: 0 (pulpa fresca) y 7 horas de secado (pulpa seca); las variables de respuesta fueron propiedades químicas y físicas. Todas las determinaciones se realizaron por triplicado. Los resultados fueron analizados mediante análisis de varianza ANOVA a un nivel de significancia del 95\%, empleando el software Minitab version demo.

\section{RESULTADOS Y DISCUSIÓN}

Los resultados referentes a la evaluación de las condiciones de secado y a la modelación matemática se relacionan a continuación, con el fin de determinar qué variables afectan el proceso, o por el contrario facilitan el mismo y a su vez establecer el modelo matemático óptimo para la predicción de los datos.

\section{Evaluación de las condiciones de secado}

Teniendo en cuenta el elevado contenido de humedad de la pulpa, se realizaron las curvas de secado con el fin de prolongar la estabilidad química y microbiológica y de esta manera establecer posibles usos a esta biomasa residual.

Con el contenido de humedad inicial de la pulpa y la masa de los sólido expresados en base seca (kg agua/ kg sólido seco) se estableció la cantidad de humedad libre para cada temperatura con relación al tiempo, (ver Figura 1). Se encontró un período de adaptación $A^{\prime}-A$ en las tres temperaturas, es decir, donde inició el proceso de calentamiento y el aumento de la temperatura de la interface. La zona A-B corresponde al primer periodo de secado y las temperaturas presentan un comportamiento similar siendo no lineal. Sin embargo, BC se puede representar como un periodo de secado constante, el cual se evidenció para las tres temperaturas, a pesar de esto, el material secado a una temperatura de $60^{\circ} \mathrm{C}$ toma un comportamiento más lineal respecto a las demás temperaturas, lo que indica que la eliminación del agua libre se realizó a una mayor tasa. En el rango C-D las líneas empiezan a formar una curvatura considerada como el punto crítico para ir constituyendo una línea asintótica, es decir donde la cantidad de agua disponible es más poca, por ende la humedad del material va reduciendo de una manera más constante hasta alcanzar el valor mínimo de humedad libre. 
El material vegetal secado a $60^{\circ} \mathrm{C}$ presenta valores más bajos de humedad los cuales son más evidentes a partir de la primera hora de secado, esto se presentó posiblemente por la mayor cantidad de energía disponible a esta temperatura para remover el agua de la superficie de la pulpa de café en un menor tiempo. Estos resultados son similares a los reportados por Pineda-Castro et al. (2009), quienes encontraron que al aumentar la temperatura del aire se incrementó la pendiente de la curva de deshidratación de la morera y disminución en el tiempo de secado. Este fenómeno es generado porque un aumento en la temperatura de secado provoca un incremento en la temperatura del producto y en el coeficiente de difusión del agua, consiguiendo que la velocidad de secado sea apreciablemente menor. A esto se suma que a mayor temperatura la humedad relativa del aire es menor, lo que aumenta su capacidad para absorber agua, favoreciendo la remoción de humedad (Bimbenet et al., 1985).

En la curva de secado se observa que algunas fases presentan un comportamiento atípico probablemente por las características intrínsecas de la pulpa de café y la resistencia de esta a la difusión interna de agua hacia la superficie en contacto con la corriente gaseosa donde logra ser evaporada (Contreras, 2006). Al final del proceso de secado se obtuvieron contenidos de humedad de $15.60,13.93$ y $13.55 \%$ para las temperaturas de 40,50 y $60{ }^{\circ} \mathrm{C}$ respectivamente (Datos no presentados). Estos resultados indican que bajo estas condiciones de secado se puede obtener el contenido de humedad similar al de hierbas para infusión comercial, que se estima en 10\% como lo reporta Velásquez-Santos y Acevedo-Álvarez, (2014).

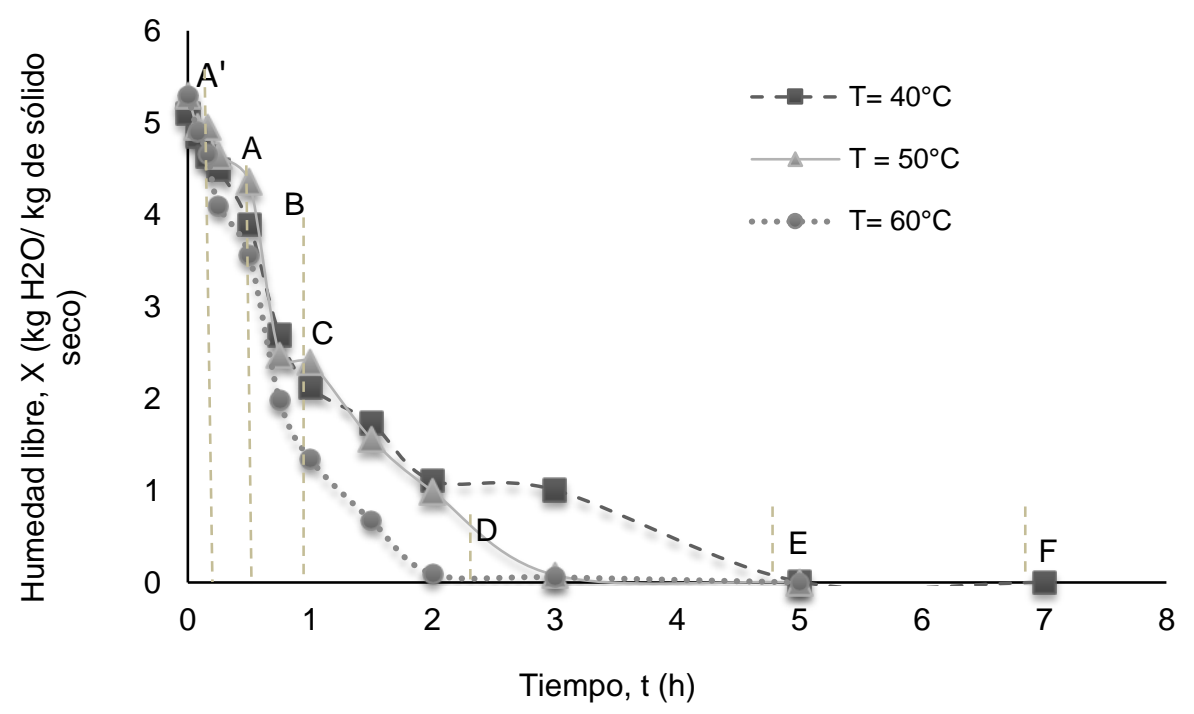

Fig.: 1 Curva de secado de la pulpa de café

\section{Velocidad de secado}

Los datos de velocidad de secado (Tabla 2), se determinaron mediante los valores obtenidos de la relación de peso de sólido seco por el área de la superficie con base en los deltas de tiempo y humedad (Ecuación 3). No se evidenció el periodo de velocidad constante para las temperaturas estudiadas solo se evidencian los cambios de velocidad decrecientes. Los mayores valores para la velocidad de secado se presentaron en la primera etapa, y una posterior disminución a medida que la humedad libre se agota. A la temperatura de $50^{\circ} \mathrm{C}$

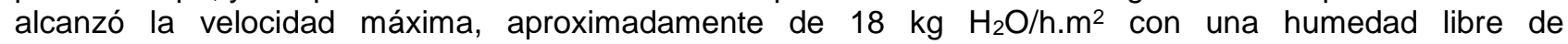
aproximadamente $4,3 \mathrm{~kg} \mathrm{H}$ 2O/kg sólido seco; a partir de la humedad libre de $3 \mathrm{~kg} \mathrm{H} \mathrm{H}_{2} \mathrm{O} / \mathrm{kg}$ sólido seco, se obtuvo una reducción de la velocidad de secado, posiblemente por la disminución de la cantidad de agua disponible, acercándose a la región de agua fuertemente ligada.

La ausencia del periodo de secado a velocidad constante puede estar asociada a que el modelo propuesto para realizar la determinación de estos valores asume un área constante dentro de la Ecuación (3), sin embargo se reporta que existe un cambio y un colapso en las estructuras del material en el proceso de deshidratación con aire caliente a causa de la remoción de agua y a los desbalances en la presión, causando encogimiento, deformación, cambios en la porosidad y fractura en algunas ocasiones por tanto no podría aplicarse a este tipo de materiales, ya que como consecuencia del secado de alimentos siempre se genera encogimiento, la estructura, tamaño y forma del producto se modifican (Nasri y Belhamri, 2018); el proceso de transferencia de calor y masa en el secado genera alteraciones físicas y químicas asociadas a la eliminación de agua del material fresco y con ello contracción y cambios de forma que a su vez influyen en la porosidad del material seco (Rizzolo et al., 2014). Por esta razón el encogimiento no debería ser despreciado 
en los estudios de secado de alimentos. Por lo anterior, se planteó la evaluación de cuatro modelos matemáticos con el objeto de predecir los datos experimentales en el proceso de secado.

Tabla 2: Velocidad de secado de la pulpa de café a 40,50 y $60^{\circ} \mathrm{C}$

\begin{tabular}{|c|c|c|c|}
\hline \multicolumn{5}{|c|}{ Velocidad de secado $\left(\mathrm{kg} \mathrm{H} \mathrm{H}_{2} \mathrm{Oh.m} \mathrm{m}^{2}\right)$} \\
\hline Tiempo $(\mathrm{h})$ & $40^{\circ} \mathrm{C}$ & $50^{\circ} \mathrm{C}$ & $60^{\circ} \mathrm{C}$ \\
\hline & $(\mathrm{n}=3)$ & $(\mathrm{n}=3)$ & $0,115 \pm 0,007$ \\
\hline 0 & $0,072 \pm 0,011$ & $0,002 \pm 0,339$ & $0,071 \pm 0,017$ \\
\hline 0,08 & $0,063 \pm 0,013$ & $0,080 \pm 0,319$ & $0,148 \pm 0,049$ \\
\hline 0,16 & $0,038 \pm 0,022$ & $0,026 \pm 0,146$ & $0,049 \pm 0,011$ \\
\hline 0,25 & $0,056 \pm 0,012$ & $0,177 \pm 0,156$ & $0,074 \pm 0,004$ \\
\hline 0,5 & $0,114 \pm 0,022$ & $0,007 \pm 0,032$ & $0,030 \pm 0,012$ \\
\hline 0,75 & $0,053 \pm 0,027$ & $0,039 \pm 0,017$ & $0,031 \pm 0,010$ \\
\hline 1 & $0,018 \pm 0,045$ & $0,027 \pm 0,009$ & $0,013 \pm 0,005$ \\
\hline 1,5 & $0,029 \pm 0,037$ & $0,021 \pm 0,001$ & $0,004 \pm 0,003$ \\
\hline 2 & $0,002 \pm 0,008$ & $0,009 \pm 0,005$ & $0,006 \pm 0,001$ \\
\hline 3 & $0,011 \pm 0,002$ & $0,002 \pm 0,003$ & $0 \pm 0$ \\
\hline 5 & $0,001 \pm 0,001$ & $0,005 \pm 0,001$ & \\
\hline 7 & $0 \pm 0$ & $0 \pm 0$ & \\
\hline
\end{tabular}

\section{Modelación matemática de la cinética de secado}

A partir de los resultados anteriores se realizó un ajuste a las curvas de secado con algunos de los modelos matemáticos de capa delgada (Tabla 1) para representar la cinética de secado de la pulpa de café a las respectivas temperaturas evaluadas.

Tabla 3: Parámetros de correlación de los modelos de secado seleccionado

\begin{tabular}{|c|c|c|c|c|c|c|c|c|c|}
\hline \multirow{3}{*}{$\begin{array}{c}\text { Modelo } \\
\text { matemático }\end{array}$} & \multicolumn{9}{|c|}{ Temperatura $\left({ }^{\circ} \mathrm{C}\right)$} \\
\hline & \multicolumn{3}{|c|}{40} & \multicolumn{3}{|c|}{50} & \multicolumn{3}{|c|}{60} \\
\hline & & $R^{2}$ & RMSE & & $R^{2}$ & RMSE & & $R^{2}$ & RMSE \\
\hline Newton & $K=1,087$ & 0,970 & 0,064 & $\mathrm{~K}=0,812$ & 0,961 & 0,067 & $K=1,224$ & 0,976 & 0,082 \\
\hline \multirow{2}{*}{ Page } & $\mathrm{n}=1,405$ & \multirow{2}{*}{0,991} & \multirow{2}{*}{0,034} & $\mathrm{n}=1,283$ & \multirow{2}{*}{0,977} & \multirow{2}{*}{0,052} & $n=1,336$ & \multirow{2}{*}{0,992} & \multirow{2}{*}{0,034} \\
\hline & $\mathrm{K}=1,180$ & & & $K=0,804$ & & & $K=1,343$ & & \\
\hline \multirow{2}{*}{$\begin{array}{c}\text { Henderson y } \\
\text { Pabis }\end{array}$} & $a=1,069$ & \multirow{2}{*}{0,977} & \multirow{2}{*}{0,056} & $a=1,048$ & \multirow{2}{*}{0,967} & \multirow{2}{*}{0,062} & $a=1,048$ & \multirow{2}{*}{0,980} & \multirow{2}{*}{0,052} \\
\hline & $\mathrm{K}=1,175$ & & & $K=0,869$ & & & $K=1,302$ & & \\
\hline \multirow{3}{*}{ Difusión } & $a=1,00$ & \multirow{3}{*}{0,970} & \multirow{3}{*}{0,064} & $a=1,00$ & \multirow{3}{*}{0,961} & \multirow{3}{*}{0,067} & $a=1,00$ & \multirow{3}{*}{0,976} & \multirow{3}{*}{0,057} \\
\hline & $b=1,00$ & & & $b=1,00$ & & & $b=1,00$ & & \\
\hline & $\mathrm{K}=1,087$ & & & $K=0,812$ & & & $K=1,224$ & & \\
\hline
\end{tabular}

A partir del coeficiente de determinación $\left(\mathrm{R}^{2}\right)$ y el error cuadrático medio (RMSE) (Tabla 3), se estableció que el mejor modelo para predecir el comportamiento de los datos experimentales fue el de Page, puesto que el coeficiente de correlación presenta un valor más alto para cada temperatura y un menor RMSE en comparación con los demás modelos evaluados, sin embargo los demás modelos también presentaron un buen ajuste. Un resultado similar fue encontrado por Lakshmi et al. (2018), quienes realizaron la modelación de las cinéticas de secado de cúrcuma blanca (Curcuma caesia) y encontraron que el modelo de Page fue el que generó mejor ajuste a los resultados experimentales. 
Los modelos cinéticos de capa delgada son muy simples comparados con las ecuaciones de secado en capas profundas, por lo cual se recomienda su uso, asociado a un menor costo computacional y con ello más facilidad de uso (Lakshmi et al., 2018).

En la Figuras 2, 3 y 4 se presentan las curvas de secado obtenidas a partir de la modelación matemática de los datos, donde se muestra la relación de humedad en función del tiempo a 40,50 y $60^{\circ} \mathrm{C}$ experimentales y modelados.

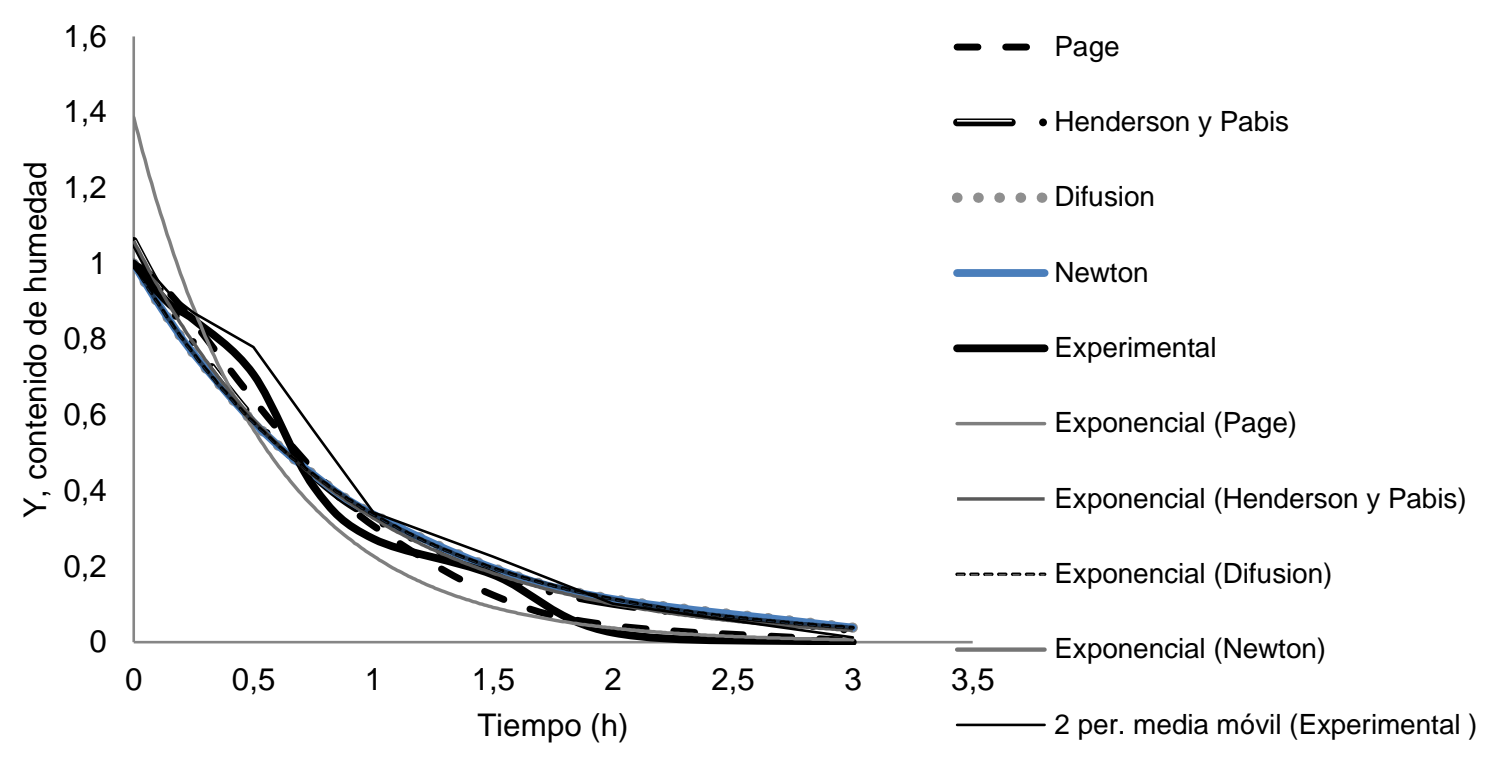

Fig. 2: Curva de secado experimental a $40^{\circ} \mathrm{C}$ para pulpa de café
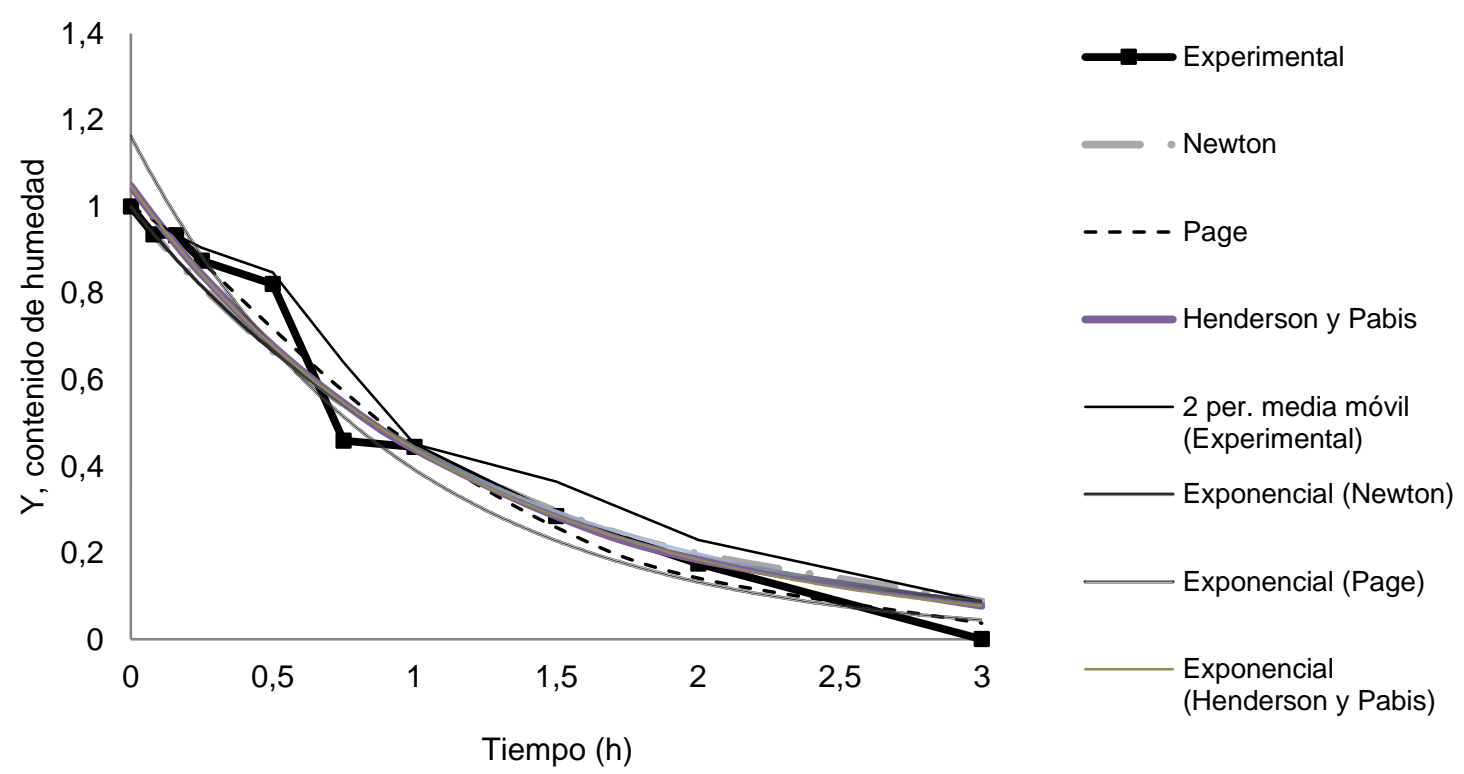

Fig.3: Curva de secado experimental a $50^{\circ} \mathrm{C}$ para pulpa de café

Se observa que, en las tres temperaturas el contenido de humedad disminuye uniformemente en función del tiempo, llegando hasta un punto asintótico; es decir que la pérdida de peso se hace notablemente lenta posiblemente porque las muestras llegaron a su peso constante. Adicionalmente, se encontró que los modelos empleados tienen un buen ajuste a los resultados experimentales, sin embargo el modelo de Page es el que mejor comportamiento presentó, lo anterior es coherente con los resultados del error cuadrático medio. 


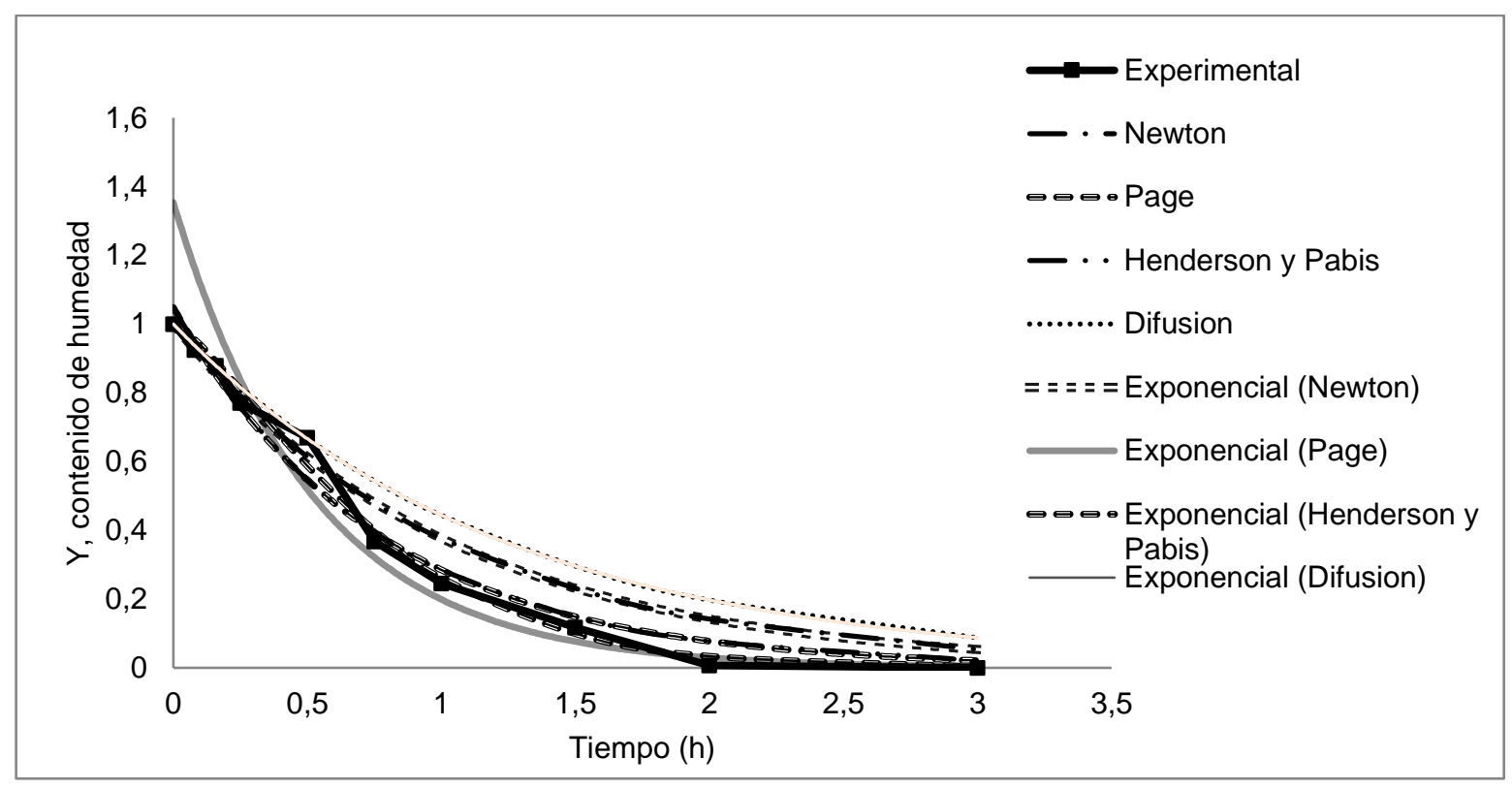

Fig. 4: Curva de secado experimental a $60^{\circ} \mathrm{C}$ para pulpa de café

\section{Propiedades químicas}

En la cuantificación de compuestos de cáscara de café húmeda y seca por cromatografía líquida de alta eficancia se evidencian dos picos a tiempo de retención de 5,1 y 6,5 minutos que corresponden a ácido clorogénico y cafeína. No se encontró presencia de ácido cafeico y ácido gálico en las pulpas evaluadas. La concentración de los compuestos se incrementó por efecto del proceso de secado, en el caso de la cafeína hubo una variación relativa con respecto a la muestra fresca del $277 \%$, pasando de 49,3 a 185,7 mg cafeína/L. Respecto al ácido clorogénico la variación fue menor (194,7\%) con valores de 22,362 y 65,912 mg ácido clorogénico / L para la pulpa fresca y húmeda.

Las variaciones encontradas pueden ser generadas por concentración de solutos durante el proceso de secado y debido a que ambos compuestos son termoestables. Wang y Lim (2015), reportan que en el café tostado se encuentra cafeína y ácido clorogénico (entre otros), adicionalmente reportan que hay un incremento en el contenido de las dos sustancias comparando el café verde y tostado. Por su parte VillalónLópez et al. (2018) encontraron los dos compuestos en granos de café tostados, lo que evidencia la estabilidad de las dos moléculas con respecto a la temperatura y una incremento asociado a menor contenido de humedad. Lo anterior muestra que estos biocomponentes no se pierden en el proceso de secado, por lo cual esta técnica es viable para estabilizar esta materia prima y desarrollar nuevos productos a partir de esta biomasa residual, con características funcionales asociadas a la cafeína y ácido clorogénico.

\section{Análisis de color}

En la Figura 5 se presentan los atributos de color en la pulpa de café antes y después del proceso de secado. Se puede observar que todos los parámetros evaluados variaron por el efecto de secado. El parámetro L* asociado a la luminosidad (relación entre blanco y negro) tuvo un incremento significativo por el proceso $(p<0.05)$, las muestras secas presentaron coloración más oscura, que pueden estar relacionadas con procesos de pardeamiento (Wang y Lim, 2015) catalizados por la temperatura empleada para el secado de la pulpa, es decir que hubo una evolución hacia un color color oscuro debido al pardeamiento (Cortés et al., 2008). Los otros parámetros tuvieron una disminución importante, excepto en el parámetro b* que no fue afectado significativamente por el proceso de secado. El parámetro $a^{*}$ (relación rojo - verde) fue mayor en la pulpa húmeda, lo cual evidencia que en las muestras durante el proceso de secado tienden a aumentar las tonalidades rojizas, por su parte el parámetro $b^{*}$ no tuvo variaciones, que pueden ser debido a que en la pulpa de café no predominan coloraciones amarillo - azul que pudieran modificarse en el secado.

El ángulo de tono $\left(\mathrm{H}^{*}\right)$ tuvo una disminución de 38 a 35\% y está ubicado en el primer cuadrante según CIELab, indicando coloraciones rojizas; la disminución del parámetro $\mathrm{H}^{*}$ indica que el color de la pulpa seca es más rojo que en la muestra fresca. Por su parte la pureza del color disminuyó, indicando que el color puede estar asociado a los diferentes valores del círculo cromático y esto puede asociarse a la formación de melanoidinas que se generan en la reacción de Maillard (Wang y Lim, 2015). El cambio total de color por efecto del proceso de secado fue de $4,8 \%$, lo que indica que los cambios anteriormente mencionados son poco perceptibles al ojo humano. 


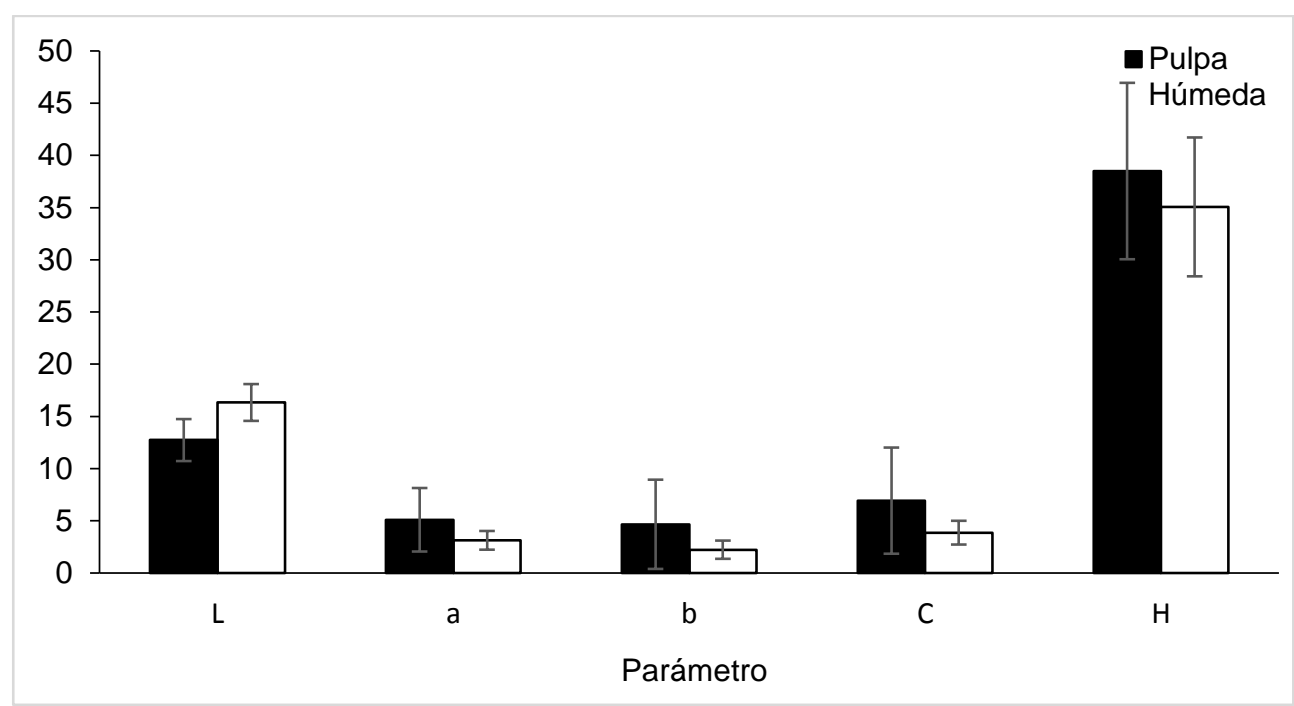

Fig. 5: Atributos de color en pulpa de café húmeda y seca, L: luminosidad, a: relación rojo - verde, b: relación amarillo - azul, C, croma, H: ángulo de tono

\section{CONCLUSIONES}

Para el secado de la cáscara de café se encontró que, las condiciones (óptimas locales) de temperatura a $60^{\circ} \mathrm{C}$ y siete horas de secado generaron el menor contenido de humedad; sin embargo, se recomienda aumentar el tiempo de secado para disminuir el contenido de humedad y obtener un producto más estable desde el punto de vista fisicoquímico en almacenamiento. La velocidad de secado presentó un comportamiento atípico donde solo se evidenciaron periodos de velocidad decreciente posiblemente por las condiciones intrínsecas del producto. Para predecir los datos experimentales a partir de la modelación matemática el mejor modelo es el de Page, sin embargo todos los modelos son adecuados para predecir el comportamiento del contenido de humedad. La pulpa de café tiene presencia de biocomponentes de alto interés (cafeína y ácido clorogénico) que no se pierden por efecto del proceso de secado y los cambios en el color son levemente percibidos por el ojo humano. Lo anterior indica que el secado es una alternativa para prolongar el tiempo de vida en almacenamiento de este subproducto.

\section{REFERENCIAS}

Armas, F.E., M.N Cornejo y Z.K. Murcia, Propuesta para el aprovechamiento de los subproductos del beneficiado del café como una alternativa para la diversificación de la actividad cafetalera y aporte de valor a la cadena productiva, Tesis de grado Ingeniería Industrial, Universidad de el Salvador, Salvador (2008)

AOAC 925.10: Association of Official Analytical Chemists (USA), Official Methods of Analysis, Determinación de humedad, 15th Ed., Vol. 1, USA (1990)

Bimbenet, J.J., J. D. Daudin y E. Wolff, Air drying kinetics of biological particles; In Toei R., Mujumdar A.S. Drying '85, pp 178-179, Berlin, Heidelberg Springer (1985)

Buntić, A.V., M.D. Pavlović y otros tres autores, Utilization of spent coffee grounds for isolation and stabilization of Paenibacillus chitinolyticus CKS1 cellulase by immobilization, doi:10.1016/j.heliyon.2016.e00146, Heliyon, 2(8) (2016)

Burdan, F., Caffeine in Coffee; Coffee in Health and Disease Prevention, 201-207 Academic Press, San Diego, USA (2015)

Cappelletti, S., D. Piacentino, G. Sani y M. Aromatario, Caffeine: Cognitive and Physical Performance Enhancer or Psychoactive Drug?, doi: 10.2174/1570159X13666141210215655'1570-159X/1875-6190, Current Neuropharmacology, 13(1), 71-88 (2015)

Ceballos-Ortiz, E.M. y M.T. Jiménez-Munguía, Cambios en las propiedades de frutas y verduras durante la deshidratación con aire caliente y susceptibilidad al deterioro microbiano,Temas selectos de ingeniería de alimentos, 6(1), 98-110 (2012)

Chu, Y.F., Y. Chen y otros seis autores, Bioactivities of crude caffeine: Antioxidant activity, cyclooxygenase-2 inhibition, and enhanced glucose uptake, doi: 2011.09.024'0308-8146, Food Chemistry, 131(2), 564-568 (2012)

Cock, L., L.S. Valenzuela y A.A. Aponte, Physical, chemical and sensory changes of refrigerated yellow pitahaya treated preharvest with 1-mcp, DYNA, 80(178), 11-20 (2013)

Contreras, M.C., Influencia del método de secado en parámetros de calidad relacionados con la estructura y el color de manzana y fresa deshidratadas, Tesis de Doctorado, Departamento de Tecnología de Alimentos, Universidad Politécnica de Valencia, España (2006)

Cortés, R.M. y A.B. Chiralt, Cinética de los cambios de color en manzana deshidratada por aire fortificada con vitamin e, doi:0121-4004, Revista de la facultad de química farmacéutica, 15(1), 8-16 (2008) 
Dadi, D., E. Mengistie y otros siete autores, Assessment of the effluent quality of wet coffee processing wastewater and its influence on downstream water quality, doi:/10.1016/j.ecohyd.2017.10.007, Ecohydrology \& Hydrobiology, 18(2), 201-211 (2018)

Fonnegra, R.G. y S.L. Jiménez, Plantas medicinales aprobadas en Colombia, $2^{\underline{a}}$ Ed., 68-72, Editorial Universidad de Antioquia, Medellín, Colombia (2007)

García-Alvarado, M.A., F.M. Pacheco-Aguirre e I.I. Ruiz-López, Analytical solution of simultaneous heat and mass transfer equations during food drying, doi: 10.1016/j.jfoodeng.2014.06.001'02608774, J. Food Eng. 142, $39-45$ (2014)

Geankoplis, C.J., Procesos de transporte y operaciones unitarias, 3ª Ed., Compañía Editorial Continental, México (1998)

Gotteland, M. y S. De Pablo, Algunas verdades sobre el café, doi: 10.4067/S0717-75182007000200002, Revista Chilena de nutrición, 34(2) (2007)

Gouvea, B.M., C. Torres y otros tres autores, Feasibility of ethanol production from coffee husks, doi:10.1007/s10529-0090023-4, Biotechnology Letters, 31(9), 1315-1319 (2009)

Hachicha, R., O. Rekik y otros seis autores, Co-composting of spent coffee ground with olive mill wastewater sludge and poultry manure and effect of Trametes versicolor inoculation on the compost maturity, doi: 10.1016/j.chemosphere.2012.03.053, Chemosphere, 88(6), 677-682 (2012)

Hall, S., B. Desbrow y otros ocho autores, A review of the bioactivity of coffee, caffeine and key coffee constituents on inflammatory responses linked to depression, doi:10.1016/j.foodres.2015.07.027'0963-9969, Food Research International, 76, 626-636 (2015)

Komes, D. y A. Bušić, Chapter 3 - Antioxidants in Coffee; Processing and Impact on Antioxidants in Beverages, pp 25-32, Academic Press, USA (2014)

Lakshmi, D.V., P. Muthukumar, A. Layek y P. Kumar, Drying kinetics and quality analysis of black turmeric (Curcuma caesia) drying in a mixed mode forced convection solar dryer integrated with thermal energy storage, doi:10.1016/j.renene.2017.12.053'0960-1481, Renewable Energy, 120, 23-34 (2018)

Mahn, A.V., C.E. Pérez y A.E. Reyes, Efecto de las Condiciones de Secado en Lecho Fluidizado Pulsante sobre el Contenido de Sulforafano de Brócoli, doi:0718-0764, Información Tecnológica, 28, 17-28 (2017)

Montes, M.E., G.R. Torres y otros cuatro autores, Modelado de la cinética de secado de ñame (Dioscorea rotundata) en capa delgada, Revista Ingeniería e Investigación, 28(2), 45-52 (2008)

Montilla-Perez, J., J. Arcila-Pulparin y otros cinco autores, Características de algunas propiedades físicas y factores de conversión del café durante el proceso de beneficio húmedo tradicional, ISSN: 0120-0275, Revista del Centro Internacional de Investigaciones de Café Cenicafé, 59(2), 120-142 (2008)

Motevali, A., S. Minaei y otros tres autores, Energy analyses and drying kinetics of chamomile leaves in microwaveconvective dryer, doi:10.1016/j.jssas.2014.11.003'1658077X, Journal of the Saudi Society of Agricultural Sciences, 15(4), 179-187 (2014)

Murthy P. y M.M. Naidu, Sustainable management of coffee industry by-products and value addition-A review, doi: 10.1016/j.resconrec.2012.06.005'0921-3449, Resources, Conservation and Recycling, 66, 45-58 (2012)

Murthy, P.S. y H.K. Manonmani, Bioconversion of Coffee Industry Wastes with White Rot Fungus Pleurotus Florida, doi:10.3923/rjes.2008.145.150, Research Journal of Environmental Sciences, 2(2), 145-150 (2008)

Nasri, M.Y. y A. Belhamri, Effects of the climatic conditions and the shape on the drying kinetics, Application to solar drying of potato-case of Maghreb's region, doi: 10.1016/j.jclepro.2018.02.103'0959-6526, Journal of Cleaner Production 183, 1241-1251 (2018)

National Academy of Science, Caffeinne for the sustainment of mental task performance: Formulations for military operations. Institute of Medicine (USA) Committee on Military Nutrition Research, National Academy Press, Washington D.C., USA (2001)

Navia, D.P., A. Ayala y H.S. Villada, Isotermas de adsorción de biolplásticos de harina de yuca moldeados por compresión, ISSN:1692-3561, Biotecnología en el Sector Agropecuario y Agroindustrial, 9(1), 77-87 (2011)

Ngo, N.T. y H.T. Phan, Screening of Fungal Strains Grown in Solid-state Culture for Production of Pectinase from Coffee Husk, doi:10.18517/ijaseit.6.3.782, International Journal on Advanced Science, Engineering and Information Technology, 6(3), 273-276 (2016)

Norhashila, H., D. Onwude y R.A. Ezdalina, Preliminary Study: Kinetic Model of Drying Process of Pumpkins (Cucurbita Moschata) in a Convective Hot Air Dryer, doi: 10.1016/j.aaspro.2014.11.048'22107843, Agriculture and Agricultural Science Procedia, 2, 345-352 (2014)

Noriega, S.A., R.A. Silva y S.M. García, Composición química de la pulpa de café a diferentes tiempos de ensilaje para su uso potencial en la alimentación animal, ISSN:0798-7269, Zootecnia Tropical 27(2), 135-145 (2009)

Novita, E., Biodegradability Simulation of Coffee Wastewater Using Instant Coffee. doi:10.1016/j.aaspro.2016.02.138, Agriculture and Agricultural Science Procedia, 9, 217-229 (2016)

Nuñez, V.A., B. Sturm y W. Hofacker, Simulation of the convective drying process with automatic control of surface temperature, doi:10.1016/j.jfoodeng.2015.08.03302608774, Journal of Food Engineering, 170, 16-23 (2016) 
Park, H.S., H.J. Lee y otros seis auotres, Effects of cosolvents on the decaffeination of green tea by supercritical carbon dioxide, doi:10.1016/j.foodchem.2007.04.064, Food Chemistry, 105(3), 1011-1017 (2007)

Pineda-Castro, M.L., V.A. Chacón y C.G. Gamboa, Efecto de las condiciones de secado sobre la cinética de deshidratación de las hojas de morera (Morus alba), doi:1021-7444, Agronomía Mesoamericana, 20(2), 275-283 (2009)

Puerta Q.G., Composición química de una taza de café, ISSN:0120-0178, Avances Técnicos, Cenicafé, 414, 1-12 (2011)

Rizzolo, A., M. Vanoli y otros diez autores, Characterizing the tissue of apple air-dried and osmo-air-dried rings by X-CT and OCT and relationship with ring crispness and fruit maturity at harvest measured by TRS, doi:10.1016/j.ifset.2013.09.0011466-8564, Innovative Food Science \& Emerging Technologies, 24, 121-130 (2014)

Rodríguez, V.N. y F.N. Zambrano, Los subproductos del café: fuente de energía renovable, ISSN: 0120-0178, Avances Técnicos, Cenicafé 393, 1-8 (2010)

Rodríguez, V.N., J.U. Sanz, C.T. Oliveros y C.G. Ramírez, Beneficio ecológico del café, Beneficio del café en Colombia, 610, Centro Nacional de Investigaciones de Café Cenicafe y Federación Nacional de Cafeteros, Chinchiná, Colombia (2015)

Ruiz-López, I.I., H. Ruiz-Espinosa, E. Herman-Lara y G. Zárate-Castillo, Modeling of kinetics, equilibrium and distribution data of osmotically dehydrated carambola (Averrhoa carambola L.) in sugar solutions. doi: 10.1016/j.jfoodeng.2010.12.01302608774, Journal of Food Engineering, 104(2), 218-226 (2011)

Toci, A.T. y M.V. Boldrin, Chapter 12 - Coffee Beverages and Their Aroma Compounds. Natural and Artificial Flavoring Agents and Food Dyes, 397-425, Academic Press, USA (2018)

Torres-Mancera, M.T., J. Cordova-López y otros cinco autores, Enzymatic extraction of hydroxycinnamic acids from coffee pulp, ISSN:1330-9862, Food Technology and Biotechnology, 49(3), 369-373 (2011)

Velásquez-Santos, C.O. y C.A. Acevedo-Álvarez, Procedimiento para deshidratación de orégano utilizando gas propano como combustible, ISSN:0128-7799, Tecno Lógicas 17(33), 13-20 (2014)

Villalón-López, N., J.I. Serrano-Contreras, D.I. Téllez-Medina y Z.L. Gerardo, An 1H NMR-based metabolomic approach to compare the chemical profiling of retail samples of ground roasted and instant coffees, doi:10.1016/j.foodres.2017.11.0770963-9969, Food Research International, 106, 263-270 (2018)

Wang, X. y L.T. Lim, Chapter 27 - Physicochemical Characteristics of Roasted Coffee. Coffee in Health and Disease Prevention, 247-254, Academic Press, San Diego, USA (2015)

Woldesenbet, A.G., B. Woldeyes y B.S. Chandravanshi, Bio-ethanol production from wet coffee processing waste in Ethiopia, doi:10.1186/s40064-016-3600-8, Springer Plus, 5(1), 1-7 (2016) 